Die Mehrwertsteuer als umweltpolitisches Lenkungsinstrument

\title{
Ermäßigung mit Tücken
}

\section{Das Verbraucherverhalten über die Höhe der Mehrwertstever in Richtung des vermehrten Konsums umweltfreundlicher Produkte zu lenken, ist keine neve \\ Idee. Realisiert wurde sie bisher nicht. $\mathrm{Ob}$ die Mehrwertstever als ergänzendes Instrument für die Fortschreibung der ökologischen Finanzreform nutzbar ist, soll eine Betrachtung des Steversystems und des rechtlichen Rahmens zeigen.}

S Von Jochen Thorwarth eit dem Jahreswechsel streitet die Republik über das neue Preissystem der Deutschen Bahn. Der Kunde möchte weniger zahlen, die Bahn mehr verdienen und die Politik mehr Bürger auf die Schiene locken. Es wundert jedoch niemanden, dass zunächst einmal die Interessen des Systembevollmächtigten, hier also die der Bahn, realisiert werden. Doch das neue Preissystem bleibt in der Kritik und dem Kundenwunsch nach niedrigeren Ticketpreisen wurde nur teilweise entsprochen. Der Bürger ist erzürnt und kehrt der Bahn den Rücken. Gibt es einen Weg, den Wünschen aller Beteiligten zu entsprechen? Abhilfe könnte die Senkung der Ticketpreise über eine Reduzierung der Mehrwertsteuer für Bahnreisen von mehr als 50 Kilometern schaffen. Das EU-Recht sieht diese Möglichkeit vor, eine Umsetzung ist jederzeit möglich. Diese nicht neue Idee fand über das Wahlprogramm von Bündnis 90/ Die Grünen Eingang in den derzeit gültigen Koalitionsvertrag der Bundesregierung, ihre Umsetzung ist für 2005 vorgesehen. Tatsächlich könnte die Förderung umweltfreundlichen Verhaltens über die Senkung der Mehrwertsteuer helfen, den Zielen aller eingangs genannten Interessengruppen näher zu kommen. Die folgende Betrachtung untersucht diesen Ansatz als einen denkbaren Baustein zur Fortschreibung der ökologischen Finanzreform.

\section{Das Instrument}

Mit dem regulären und zwei ermäßigten Steuersätzen existiert bereits ein erprobtes Instrument zur politischen Einflussnahme über die Mehrwertsteuer. Der Einsatz des Instrumentes ist aus verteilungspolitischen Gründen (Lebensmittel), auf Grund ökonomischer (Rohstoffe) oder auch kulturpolitischer Motive (Printmedien) zulässig und etabliert.

Außer der geltenden ermäßigten Besteuerung des öffentlichen Personennahverkehrs beinhaltet das deutsche Mehrwertsteuersystem jedoch keine nennenswerten Regelungen mit ökologischer Wirkung. Und auch diese Begünstigung ist eher sozial- als umweltpolitisch motiviert.

Auf den ersten Blick erscheint es sinnvoll, die Möglichkeiten der Mehrwertsteuer zur Erweiterung des umweltpolitischen Instrumentariums zu nutzen. Sie erreicht als allgemeine Verbrauchssteuer mehr oder weniger alle Produktund Dienstleistungsgruppen. Mit Hilfe Anreize schaffender Steuersatzstaffeln träfe man gezielt das Verbraucherverhalten in ausgewählten, besonders umweltrelevanten Produktgruppen. So zieht das EU-Grünbuch zur Integrierten Produktpolitik (IPP) die Mehrwertsteuer als eine Option zur Verbesserung der Wettbewerbsfähigkeit ökologisch vorteilhafter Produkte in Betracht (1).

\section{Rechtliche Grundlagen}

Der rechtliche Rahmen der Mehrwertsteuer wird in Brüssel abgesteckt. Die europäische Mehrsteuerrichtlinie lässt den Mitgliedstaaten nur einen engen Spielraum zur nationalen Gestaltung des Systems (2).

Die Richtlinie setzt auf Grund der unterschiedlichen Bedeutung der Mehrwertsteuereinnahmen für die EU-Haushalte nur Untergrenzen für die Höhe der Steuer, nach oben sind keine Grenzen gesetzt. Der reguläre Satz darf 15 Prozent nicht unterschreiten, die beiden zulässigen ermäßigten Sätze müssen mindestens 5 Prozent betragen. Deshalb schwankt die Höhe des regulären Satzes EU-weit zwischen 15 und 25 Prozent Manche Staaten erheben einen, im Fall Deutschland 7 Prozent, andere beide zulässigen Ermäßigungssätze.

Welche Produkte und Dienstleistungen ermäßigt besteuert werden dürfen, legt Anhang $\mathrm{H}$ der Richtlinie fest. Auf Grundlage dieser erschöpfenden Liste entscheiden die Einzelstaaten, welche ermäßigten Steuergegenstände sie in das natio- nale System übernehmen. Solche mit ökologischer Wirkung finden sich dort, den öffentlichen Personenverkehr ausgenommen, nicht (3).

Zur Erweiterung des Anhangs bedarf es des einstimmigen Beschlusses des EU-Finanzministerrates - eine denkbar hohe Hürde, wenn die Liste um umweltorientierte Inhalte ergänzt werden soll! Für eine Modifizierung der Mehrwertsteuer nach ökologischen Kriterien muss der Weg über Brüssel gehen. Dies ist nur unter Einhaltung der europäischen Binnenmarktregeln vorstellbar. Insbesondere muss sichergestellt werden, dass die bevorzugte Behandlung einzelner, ökologisch vorteilhafter Produkte nicht den Charakter einer unzulässigen Beihilfe für bestimmte Branchen oder Unternehmen annimmt. Um dies zu gewährleisten, muss die Einstufung der ausgewählten Produkte streng anhand von Kriterien, die sich an objektiven Produkteigenschaften orientieren, erfolgen. Auch die willkürliche Diskriminierung von Produkten aus den Partnerländern ist verboten. Die Herkunft des Produktes darf deshalb kein Argument für die Einstufung in die Steuerklassen sein.

Von ähnlicher Bedeutung ist die Frage, ob die Aufnahme des Umweltschutzes als Lenkungsziel das Neutralitätsprinzip der Mehrwertsteuer, also die Vorgabe alle Umsätze gleichermaßen zu belasten, überstrapaziert. Die eingangs genannten Beispiele für Steuerermäßigungen aus sozialen, ökonomischen und kulturellen Gründen werden mit einem gesamtgesellschaftlichen Interesse begründet. Doch spätestens seit Einführung der ökologischen Steuerreform gelten ökologische Aspekte ebenfalls als außerfiskalische Ziele der deutschen Steuerpolitik.

\section{Perspektiven in der EU}

Die Position der EU-Kommission ist widersprüchlich. Im Zusammenhang mit der IPP wird der Mehrwertsteuer für die Zukunft eine bedeutende Rolle für den Umweltschutz zugesprochen. An anderer Stelle empfiehlt sie, nach Möglichkeit auf weitere Ausnahmen vom Regelsatz zu verzichten und eventuell sogar deren völlige Abschaffung in Betracht zu ziehen (3).

Der aktuellste Bezug findet sich bei einem zur Zeit laufenden EU-Pilotprojekt zur Bekämpfung der Schwarzarbeit im Bereich gering bezahlter Dienstleistungen. Um einen Anreiz zu schaffen, die Umsätze aus solchen Tätigkeiten wieder regulär zu versteuern und die Entstehung neuer Arbeitsplätze in diesem Segment zu stimulieren, ist es den Mitgliedsstaaten erlaubt, auf Dienstleistungen wie 
zum Beispiel Fahrradreparaturen oder Reinigungsarbeiten den ermäßigten Steuersatz anzuwenden. Die reduzierte Belastung soll helfen, die durch hohe Personalkosten bedingte Unwirtschaftlichkeit zu mildern. Zahlreiche Staaten machen derzeit von dieser Möglichkeit Gebrauch. Deutschland nimmt an dem Versuch nicht teil.

Von den Ergebnissen dieses Versuches macht die EU-Kommission abhängig, ob die Einführung weiterer, unter anderem ökologisch begründeter Steuerermäßigungen in Betracht kommt (4). Das Projekt wurde wiederholt verlängert, zuletzt bis Ende 2003. Die Kommission erwägt bereits eine dauerhafte Übernahme des Modells in das europäische Mehrwertsteuerrecht (5).

Neben den rechtlichen und politischen Hürden, die Brüssel für die Idee einer Mehrwertsteuer mit ökologischer Komponente darstellt, ist die EUübergreifende Entwicklung des Systems aber auch eine Chance. Berücksichtigt die weitere Harmonisierung auch ökologische Aspekte, wäre eine weitreichende Wirksamkeit der Maßnahme gewiss.

\section{Anforderungen an eine Reform}

Zur Gewährleistung der Wirksamkeit einer Mehrwertsteuer mit ökologischer Komponente müssen folgende Anforderungen an denkbare Modelle gestellt werden:

- Der durch die Anwendung des ermäßigten Satzes entstandene Preisvorteil muss für den Verbraucher deutlich erkennbar sein - sonst ist eine lenkende Wirkung etwa für eine breite Produktpalette mit einer großen Preisspanne nicht zu erwarten.

- Die Weitergabe der Ermäßigung durch den Verkäufer an den Kunden muss sichergestellt werden. Andernfalls käme es lediglich zu einer Verbreiterung der Marge für den Händler.

- Klare Umweltqualitätsziele ermöglichen eine sinnvolle Beurteilung der Wirksamkeit von Lenkungsmaßnahmen. Dazu könnten in Anlehnung an die Vergabekriterien für Umweltzeichen klar definierte Anforderungen für die ermäßigte Veranlagung formuliert werden.

- Um das Funktionieren des Systems zu garantieren und um unverantwortbaren Aufwand zu vermeiden, sollte die Anzahl der gewährten Ausnahmen vom Regelsatz gering gehalten werden. So wird den berechtigten Warnungen vor einem administrativem Kollaps des deutschen Steuersystems Rechnung getragen. Auch für Unternehmen mit einer breiten Produktpalette wird der Verwaltungsaufwand mit zunehmender Anzahl von Ausnahmen unzumutbar groß.
- Außerdem sollte darauf verzichtet werden, Umweltanforderungen an das Herstellungsverfahren von Produkten zu stellen. Der damit verbundene Kontrollaufwand, insbesondere bei ausländischen Gütern, wäre kaum zu bewältigen.

\section{Fazit}

Mit zwei Ermäßigungssätzen stehen bereits erprobte Lenkungsinstrumente zur Verfügung. Aus umweltpolitischer Sicht interessante Produkte und Dienstleistungen werden bei der gegenwärtigen Zusammensetzung der Ermäßigungsliste mit Ausnahme des öffentlichen Personenverkehrs jedoch nicht erreicht. Da eine kurzfristige Änderung des Anhanges wegen des konsensuellen Verfahrens nicht zu erwarten ist, sollte im Zuge der europäischen Harmonisierung der Mehrwertsteuerregeln auf eine Integration ökologisch sinnvoller Komponenten hingewirkt werden. Zunächst einmal bleibt abzuwarten, ob der reduzierte Steuersatz im Schienenfernverkehr kommt und welche Effekte davon ausgehen. Die ÖkoMehrwertsteuer ist kein Instrument für den breiten Einsatz. In ausgewählten Einzelfällen kann sie das ökologisch-ökonomische Instrumentarium sinnvoll ergänzen (6).

\section{Anmerkungen}

(1) Grünbuch zur integrierten Produktpolitik, KOM (2001)

68; Brüssel den 7.2.2001, S.11.

(2) EU-Richtlinie 77/388/EWG zur Harmonisierung der Mehrwertsteuer; in der konsolidierten Fassung

1977L0388, Brüssel den 15.5.2002.

(3) Mitteilung der EU-Kommission, KOM (2001) 599 endgültig; S. 14 Punkt 58; Brüssel den 22.10.2001.

(4) Mitteilung der EU-Kommission, KOM(2000)348 endgültig; S.16; Brüssel den 07.06.2000.

(5) Richtlinie des Rates 2002/92/EG vom 3. Dezember 2002, Amtsblatt der Europäischen Gemeinschaften L $331 / 27,7.12 .2002$.

(6) Grundlage des Artikels ist die Abschlussarbeit Die Mehrwertstever als umweltpolitisches Lenkungsinstrumentrechtliche Grundlagen und denkbare Modelle, entstanden im Rahmen des Aufbaustudienganges "Umwelt- und Qualitätsmanagement" an der Fachhochschule für Wirtschaft, Berlin, 2002.

\section{Der Autor}

Jochen Thorwarth ist VÖW-Mitglied.

Kontakt: Schönleinstr. 25,10967 Berlin,

Tel. 030-6933437

E-Mail: jochen.thorwarth@gmx.de

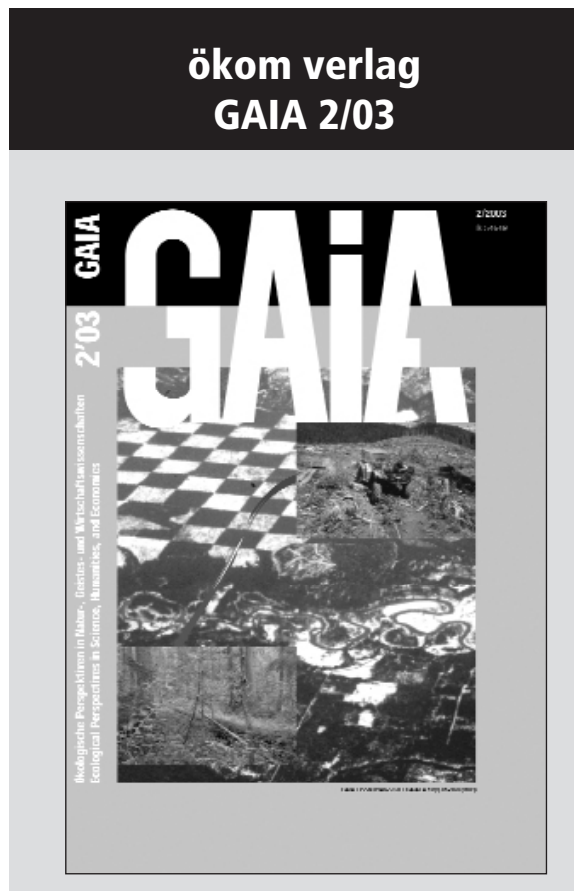

Wie misst man die Qualität von Wissenschaft? Warum stößt das klassische Modell der Evaluation an seine Grenzen, wenn es gilt, außeruniversitäre Forschung, inter- und transdisziplinäre Forschung zu bewerten? Und welche Art der Evaluation braucht diese Forschung? Hansvolker Ziegler, Ernst Ulrich von Weizsäcker, Hartmut Graßl, Franz Josef Radermacher, Lenelis Kruse und Walter Peissl gehen diesen Fragen im GAlA-Disput auf den Grund.

In GAIA 2/03 lesen Sie außerdem:

-Wie lässt sich die politische Förderung des Ökolandbaus begründen? (Beiträge von Stephan Dabbert und Anna Maria Häring; Stefan Mann; Ulrich Koester)

- Integrative Biodiversity Research Emerging Issues for Linking Natural and Social Sciences (Anke Jentsch et al.)

Abonnement von 4 Ausgaben: Einzelpersonen: 79,- $€$,

Institutionen: 139,- $€$, Studenten: 50,- $€$ Einzelheft: $20,-€$ (zzgl. Versandkosten) GAIA ist jetzt online bei

www.ingentaselect.com

www.gaia-online.net

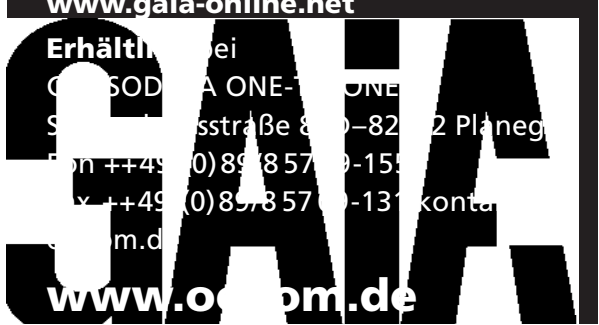


(c) 20I0 Authors; licensee IÖW and oekom verlag. This is an article distributed under the terms of the Creative Commons Attribution Non-Commercial No Derivates License (http://creativecommons.org/licenses/by-nc-nd/3.o/), which permits unrestricted use, distribution, and reproduction in any medium, provided the original work is properly cited. 